\title{
Tigeciclina:
}

\section{rassegna della letteratura}

Mario Venditti ${ }^{(1)}$, Maria Elena Pompeo ${ }^{(1)}$, Flavia Fabi ${ }^{(1)}$

REVIEW

\begin{abstract}
Tigecycline is a new first-in-class glycylcycline antimicrobial agent with expanded broad-spectrum activity. It was developed to overcome the two key resistance mechanisms, efflux pumps and ribosomal protection, that limit the use of tetracycline.

The spectrum of activity extends to clinically relevant susceptible and multidrug resistant bacteria, as methicillin resistant Staphylococcus aureus (MRSA), Streptococcus pneumoniae, vancomycin resistant Enterococci, Acinetobacter spp, Acinetobacter baumannii and Enterobacteriaceae, including extended-spectrum $\beta$-lactamase-producing strains (ESBLs).

Tigecycline has been introduced into clinical practice as part of the effort to combat the growing problem of bacterial resistance to anti-infective therapy: tigecycline could replace some broad-spectrum agents for approved indications reducing the selective pressure provided by antibiotic administration.

The expanded in vitro activity against a broad range of bacteria, including resistant pathogens, of tigecycline suggest that this novel antimicrobial agent should offer clinicians an option for the treatment of patients with serious bacterial infections.
\end{abstract}

Keywords: Tigecycline, glycylcycline, broad-spectrum antibiotics, bacterial infections Farmeconomia e percorsi terapeutici 2006; 7(4): 211-222

\section{INTRODUZIONE}

La necessità di nuovi agenti antibatterici nella pratica clinica è molto sentita a causa dell'insorgenza di nuove infezioni e dello sviluppo di resistenze multifarmaco da ceppi batterici responsabili di patologie gravi e a rischio di vita.

Le resistenze batteriche spesso rendono inappropriate o inadeguate le attuali terapie, determinando fallimenti nel trattamento con conseguente aumento dei costi, della morbilità e della mortalità.

Particolare preoccupazione deriva dai ceppi di Staphylococcus aureus meticillino-resistenti (MRSA), di S. aureus a resistenza intermedia verso i glicopeptidi (GISA) e di Enterococco vancomicino-resistente (VRE). Inoltre stanno emergendo resistenze tra $\mathrm{i}$ gram-negativi nei confronti di tutti gli agenti antimicrobici approvati.

In Europa si è verificato un incremento degli organismi patogeni resistenti; molti di questi, come ad esempio l'MRSA, hanno sviluppato una resistenza a diversi antibiotici [1].

I pazienti ospedalieri, come risultato di un sistema immunitario spesso indebolito, sono particolarmente vulnerabili nei confronti di questi ceppi resistenti [2], sviluppando infezioni che possono manifestarsi, tra l'altro, come ustioni infette, ascessi profondi, infezioni da ferite chirurgiche, incluse quelle derivanti da complicanze dei grossi interventi sul tratto gastroenterico (perforazioni intestinali, deiscenze delle anastomosi).

Tali complicazioni cliniche, in combinazione con le condizioni mediche preesistenti dei pazienti, possono rivelarsi fatali o portare a prolungate degenze in ospedale, richiedendo di conseguenza ulteriori cure sanitarie [3]. Quando un paziente ospedaliero manifesta una grave infezione si impiegano tra le 24 e le 48 ore per individuare i batteri responsabili. Può essere dunque di vitale importanza, negli stadi iniziali critici del trattamento, avere a disposizione antibiotici a largo spettro che risultino efficaci.

In questo panorama si inserisce tigeciclina, capostipite delle glicilglicine, una nuova categoria di antibiotici a largo spettro derivata dalle tetracicline. Tigeciclina è stata specificamente sviluppata per superare i principali meccanismi di resistenza delle tetracicline che attualmente ne limitano fortemente le possibilità di utilizzo. Nonostante il bisogno di nuovi agenti in grado di superare efficacemente i meccanismi di resistenza sia divenuto cruciale, lo sviluppo di tali agenti è decisamente in declino, al punto che la FDA ha garantito a tigeciclina un fast track approval.

\author{
${ }^{(1)}$ Dipartimento di \\ Medicina Clinica, \\ Università \\ "La Sapienza"
}




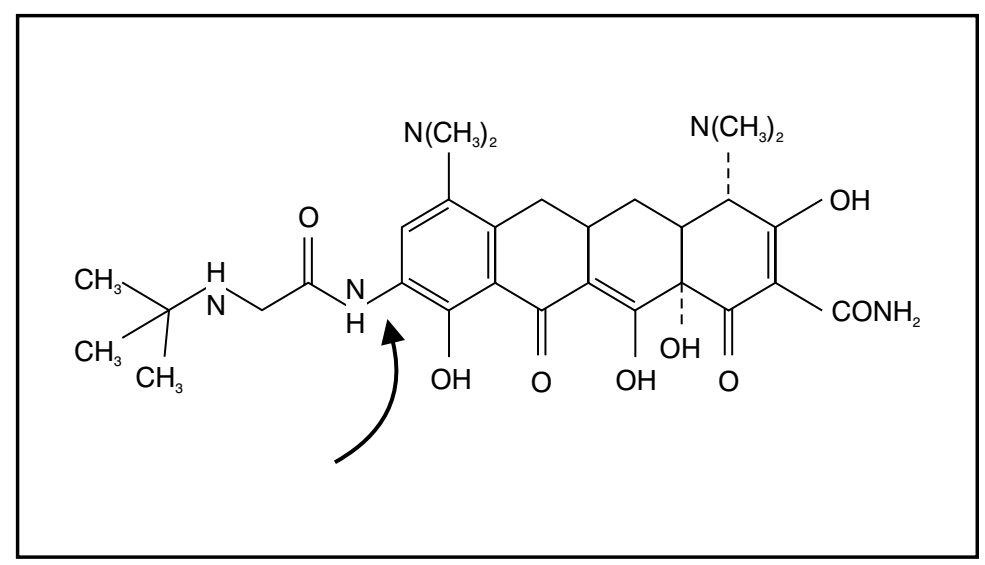

Figura 1

Struttura della tigeciclina cinque volte superiore alle tetracicline [7], dall'altro impedisce il trasporto attraverso le pompe di efflusso.

Inoltre, tigeciclina sfugge ad altri meccanismi di resistenza come la produzione di $\beta$-lattamasi a spettro esteso (ESBL, Extended Spectrum $\beta$-Lactamases) che può limitare la scelta degli antibiotici disponibili [8].

\section{STUDI IN VITRO}

L'attività in vitro di tigeciclina è stata testata su un gran numero di agenti patogeni nei due grandi studi TEST (Tigecycline Evaluation Surveillance Trial) $[9,10]$ e SENTRY [11-13], i quali mostrano che l'antibiotico presenta valori

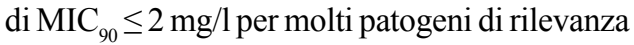
clinica, in particolare per quelli implicati nelle infezioni complicate della cute e dei tessuti molli (complicated Skin and Skin Structure Infections, cSSSI) e nelle infezioni complicate intra-addominali (complicated Intra-Abdominal Infections, cIAI).

\section{Attività in vitro contro gram-positivi}

Tigeciclina ha mostrato attività contro più di 20.000 ceppi patogeni di $S$. aureus oxacillinosensibili e oxacillino-resistenti, con $\mathrm{MIC}_{90}=0,5$ $\mathrm{mg} / \mathrm{l}$. Inoltre tigeciclina inibisce batteriostaticamente anche ceppi di $S$. aureus vancomicino-resistenti, presentandosi come una valida opzione terapeutica per ceppi di $S$. aureus resistenti a più classi di antibiotici. È inoltre altamente attiva contro stafilococchi coagulasi-negativi, inclusi ceppi meticillino-resistenti, con valori di $\mathrm{MIC}_{90}$ compresi tra 0,5 e $1 \mathrm{mg} / \mathrm{l}$ e ha dimostrato eccellente attività contro molti streptococchi, incluso Streptococcus pneumoniae a ridotta sensibilità o resistenza verso macrolidi e antibiotici $\beta$-lattamici. Tigeciclina mostra una potenza maggiore, rispetto agli altri antibiotici testati, contro enterococchi vancomicino-sensibili e vancomicinoresistenti, con valori di $\mathrm{MIC}_{90}$ compresi tra 0,25 e $0,5 \mathrm{mg} / \mathrm{l}$. In Tabella I si riporta l'attività in vitro di tigeciclina contro i gram-positivi.

\section{Attività in vitro contro gram-negativi}

Tigeciclina è altamente attiva contro gli enterobatteri e il $99 \%$ dei ceppi è inibito da concentrazioni $\leq 4 \mathrm{mg} / \mathrm{l}$. Attività ridotta è stata osservata nei confronti di Proteus mirabilis e Pseudomonas aeruginosa in quanto è vulnerabile alle pompe di efflusso multifarmaco cromosomicamente codificate presenti in queste specie. Nei confronti di Stenotrophomonas maltophilia tigeciclina ha mostrato attività superiore a quella di amikacina, ceftazidima e ticarcillina + clavulanato [15].

Tigeciclina ha dimostrato buona attività anche contro altri batteri gram-negativi non fermentanti, come Acinetobacter spp. (il 90\% dei ceppi è in genere sensibile) e Acinetobacter 
baumannii (92\% dei ceppi), risultando anche attiva su ceppi resistenti all'imipenem [16].

Infine ha una potente attività in vitro contro due patogeni comunemente isolati nel tratto respiratorio, Haemophilus influenzae e Moraxella catarrhalis, sia in assenza che in presenza di $\beta$ lattamasi. L'attività in vitro di tigeciclina contro i gram-negativi è riassunta in Tabella II.

\section{Attività in vitro contro batteri anaerobi}

Tigeciclina ha attività maggiore di clindamicina, minociclina, trovafloxacina e cefoxitina contro Bacteroides fragilis [17]. Ha inoltre dimostrato eccellente attività contro molti peptostreptococchi, con $\mathrm{MIC}_{90} \leq 4 \mathrm{mg} / 1$ [18] e contro Clostridium difficilis (il $92,7 \%$ dei ceppi risulta inibito a concentrazioni di 0,25 mg/l) [19] (Tabella III).

\section{Attività in vitro contro microrganismi atipici}

Tigeciclina ha un'attività maggiore contro Mycoplasma pneumoniae rispetto a minoci- clina e tetracicline, con $\mathrm{MIC}_{90}=0,25 \mathrm{mg} / \mathrm{l}$. Tigeciclina è molto attiva contro i micobatteri non tubercolari [20] e contro molte specie di Chlamydophila [21] (Tabella IV).

Il dato che emerge dal confronto dei dati degli studi in vitro condotti su tigeciclina (Tabella V) è la maggiore ampiezza di spettro antibatterico del farmaco rispetto agli altri antibiotici con cui è stata confrontata.

\section{FARMACOCINETICA}

\section{Assorbimento}

Tigeciclina è somministrata per via endovenosa e quindi ha il $100 \%$ di biodisponibilità.

\section{Distribuzione}

Il legame di tigeciclina alle proteine plasmatiche in vitro varia dal $71 \%$ circa all' $89 \%$, alle concentrazioni osservate negli studi clinici (da 0,1 a $1,0 \mu \mathrm{g} / \mathrm{ml}$ ). Studi di farmacocinetica negli animali e nell'uomo hanno dimostrato

\begin{tabular}{|c|c|c|c|}
\hline Organismo & $\begin{array}{c}\text { MIC range } \\
(\mathrm{mg} / \mathrm{l})\end{array}$ & $\begin{array}{c}\text { MIC }_{50} \text { range } \\
(\mathrm{mg} / \mathrm{l})\end{array}$ & $\begin{array}{c}\mathrm{MIC}_{90} \text { range } \\
(\mathrm{mg} / \mathrm{l})\end{array}$ \\
\hline Staphylococcus aureus & $\leq 0,02-2$ & $0,06-0,5$ & $0,125-1$ \\
\hline S. aureus $\left(\mathrm{OXA}^{\mathrm{S}}\right)$ & $0,06-1$ & $\leq 0,13-0,5$ & $0,25-0,5$ \\
\hline S. aureus $\left(\mathrm{OXA}^{\mathrm{R}}\right)$ & $\leq 0,06-2$ & $\leq 0,13-0,5$ & $0,25-1$ \\
\hline S. aureus (VAN') & $0,06-2$ & 0,25 & 0,5 \\
\hline Staphylococcus coagulasi negativo & $\leq 0,03-2$ & $0,06-1$ & $0,25-1$ \\
\hline CNS $\left(O_{X} A^{S}\right)$ & $\leq 0,03-1$ & $0,25-0,5$ & $0,25-1$ \\
\hline CNS $\left(O X A^{R}\right)$ & $\leq 0,03-2$ & $0,5-1$ & $0,25-1$ \\
\hline Enterococcus species & $\leq 0,02-2$ & $0,03-0,25$ & $0,06-0,5$ \\
\hline E. faecalis & $\leq 0,02-2$ & $0,13-0,25$ & $0,13-0,5$ \\
\hline E. faecalis (VANR) & $\leq 0,03-0,5$ & 0,13 & $0,13-0,5$ \\
\hline E. faecium & $\leq 0,03-0,5$ & $0,06-0,25$ & $0,13-0,25$ \\
\hline E. faecium (VAN') & $\leq 0,03-0,5$ & $0,06-0,13$ & 0,13 \\
\hline E. avium & $0,06-0,13$ & 0,06 & 0,06 \\
\hline E. casseliflavus & $\leq 0,03-0,5$ & $0,13-0,25$ & $0,13-0,25$ \\
\hline E. gallinarum & $0,06-2$ & 0,13 & $0,13-0,25$ \\
\hline E. raffinosus & $0,06-0,5$ & 0,06 & 0,13 \\
\hline Streptococcus pneumoniae & $\leq 0,01-1$ & $\leq 0,02-0,25$ & $\leq 0,02-0,5$ \\
\hline S. pneumoniae $\left(\mathrm{PEN}^{\mathrm{S}}\right)$ & $\leq 0,02-0,5$ & $0,03-0,25$ & $0,13-0,25$ \\
\hline S. pneumoniae (PEN') & $\leq 0,02-1$ & $0,03-0,25$ & $0,06-0,5$ \\
\hline S. pneumoniae (PENR) & $\leq 0,02-1$ & $0,06-0,25$ & $0,13-0,25$ \\
\hline S. pneumoniae (TETs) & $0,01-0,13$ & 0,03 & 0,03 \\
\hline S. pneumoniae $\left(\mathrm{TET}^{\mathrm{R}}\right)$ & $0,02-0,5$ & 0,03 & 0,03 \\
\hline Streptococci gruppo A & $\leq 0,02-0,5$ & $0,06-0,13$ & $0,06-0,25$ \\
\hline Streptococci gruppo B & $0,03-0,5$ & $0,06-0,13$ & $0,06-0,25$ \\
\hline Viridans streptococci & $0,01-2$ & $\leq 0,02-0,06$ & $0,03-0,5$ \\
\hline Viridans streptococci (PEN $)$ & $0,03-0,25$ & 0,06 & 0,25 \\
\hline Viridans streptococci (PEN') & $0,02-0,13$ & 0,03 & 0,06 \\
\hline Viridans streptococci (TETs) & $0,02-0,06$ & 0,03 & 0,06 \\
\hline Viridans streptococci (TET) & $0,1-0,5$ & 0,06 & 0,13 \\
\hline
\end{tabular}

\section{Tabella I}

Attività in vitro di tigeciclina contro gram-positivi (modificato da [14])

$\mathrm{CNS}=$

Staphylococcus coagulasi negativo I = Intermedio OXA $=$ Oxacillina $\mathrm{PEN}=$ Penicillina $\mathrm{R}=$ Resistente $\mathrm{S}=$ Suscettibile TET = Tetraciclina VAN $=$ Vancomicina 


\section{Tabella II}

Attività in vitro di tigeciclina contro gramnegativi (modificato da [14])

$\mathrm{CIP}=$ Ciprofloxacina

$\mathrm{ESBL}=$ Extendedspectrum Betalactamase

$\mathrm{I}=$ Intermedio

$\mathrm{R}=$ Resistente

$\mathrm{S}=$ Suscettibile

$\mathrm{TET}=$ Tetraciclina

\begin{tabular}{|c|c|c|c|}
\hline Organismo & $\begin{array}{c}\text { MIC range } \\
(\mathrm{mg} / \mathrm{l})\end{array}$ & $\begin{array}{c}\text { MIC }_{50} \text { range } \\
(\mathrm{mg} / \mathrm{l})\end{array}$ & $\begin{array}{c}\mathrm{MIC}_{90} \text { range } \\
(\mathrm{mg} / \mathrm{l})\end{array}$ \\
\hline Escherichia coli & $0,06-2$ & $0,13-0,5$ & $0,25-1$ \\
\hline E. coli (non-ESBL) & $0,06-2$ & $0,13-0,5$ & $0,25-1$ \\
\hline E. coli (ESBL) & $0,06-2$ & $0,13-0,25$ & $0,5-1$ \\
\hline E. coli (CIPs) & $0,5-2$ & 1 & 1 \\
\hline E. coli (CIPR) & $0,5-2$ & 1 & 1 \\
\hline Klebsiella pneumoniae & $0,06-8$ & $0,25-1$ & $1-2$ \\
\hline K. pneumoniae (non-ESBL) & $0,06-4$ & $0,25-1$ & $1-2$ \\
\hline K. pneumoniae (ESBL) & $0,06-8$ & $0,25-1$ & $1-2$ \\
\hline Klebsiella oxytoca & $0,5-2$ & $0,5-1$ & 1 \\
\hline Morganella morganii & $1-8$ & $2-4$ & 4 \\
\hline Proteus mirabilis & $1-8$ & 4 & 8 \\
\hline Proteus vulgaris & $0,13-0,16$ & 4 & 4 \\
\hline Providencia species & $4-8$ & 4 & 8 \\
\hline Shigella species & $0,13-0,5$ & 0,25 & 0,5 \\
\hline Salmonella species & $0,25-2$ & 1 & 1 \\
\hline Citrobacter species & $0,25-16$ & $0,5-1$ & $0,5-2$ \\
\hline C. freundii & $0,25-16$ & $0,5-1$ & 2 \\
\hline Enterobacter species & $0,25-8$ & 1 & $1-2$ \\
\hline E. cloacae & $0,25-4$ & 1 & 2 \\
\hline E. aerogenes & $0,25-8$ & 1 & 1 \\
\hline Serratia marcescens & $0,5-8$ & $2-4$ & $2-4$ \\
\hline Stenotrophomonas maltophilia & $0,25-8$ & $0,5-2$ & $2-4$ \\
\hline Pseudomonas aeruginosa & $0,5-32$ & $8->16$ & $16-32$ \\
\hline Acinetobacter species & $\leq 0,03-16$ & $0,25-2$ & $0,5-8$ \\
\hline A. baumannii & $0,03-16$ & $0,5-2$ & $2-8$ \\
\hline Burkholderia cepacia & $0,5-64$ & $2-4$ & $4-32$ \\
\hline Haemophilus influenzae & $\leq 0,13-4$ & $0,25-1$ & $0,5-2$ \\
\hline Moraxella species & $\leq 0,03-0,25$ & $0,06-0,13$ & $0,13-0,25$ \\
\hline M. catarrhalis & $\leq 0,03-2$ & $0,06-0,13$ & $0,13-0,5$ \\
\hline Neisseria gonorrhoeae & $\leq 0,02-1$ & $0,06-0,5$ & $0,13-1$ \\
\hline N. gonorrhoeae (TETs) & $\leq 0,02-0,25$ & 0,06 & 0,13 \\
\hline N. gonorrhoeae (TET') & $0,06-0,25$ & 0,13 & 0,25 \\
\hline N. gonorrhoeae (TETR) & $0,06-1$ & 0,25 & 0,5 \\
\hline Eikenella corrodens & $\leq 0,06-4$ & 0,5 & 2 \\
\hline
\end{tabular}

\begin{tabular}{lccc}
\hline \multicolumn{1}{c}{ Organismo } & $\begin{array}{c}\text { MIC } \text { range } \\
(\mathbf{m g} / \mathbf{l})\end{array}$ & $\begin{array}{c}\mathbf{M I C}_{\mathbf{5 0}} \text { range } \\
\mathbf{( m g / l )}\end{array}$ & $\begin{array}{c}\mathbf{M I C}_{\mathbf{9 0}} \text { range } \\
\mathbf{( m g / l )}\end{array}$ \\
\hline Bacteroides fragilis & $0,5-8$ & 2 & 2 \\
Bacteroides fragilis gruppo & $0,02-2$ & $0,13-0,5$ & $0,13-2$ \\
Clostridium perfringens & $0,03-4$ & $0,03-0,5$ & $0,25-1$ \\
Clostridium difficile & $\leq 0,02-0,25$ & $0,03-0,13$ & $0,03-0,13$ \\
Proprionibacterium acnes & $0,03-0,13$ & 0,03 & 0,06 \\
Peptostreptococcus species & $\leq 0,02-0,5$ & $0,03-0,06$ & $0,03-0,25$ \\
Fusobacterium species & $\leq 0,02-0,25$ & $0,02-0,06$ & 0,06 \\
Prevotella species & $0,02-1$ & $0,03-0,5$ & $0,06-1$ \\
Porphyromonas species & $\leq 0,02-0,13$ & $0,03-0,06$ & 0,06 \\
\hline
\end{tabular}

\section{Tabella III}

Attività in vitro di

tigeciclina contro

anaerobi (modificato da [14]) 


\begin{tabular}{|c|c|c|c|}
\hline Organismo & $\begin{array}{c}\text { MIC range } \\
(\mathrm{mg} / \mathrm{l})\end{array}$ & $\begin{array}{c}\mathrm{MIC}_{50} \text { range } \\
(\mathrm{mg} / \mathrm{l})\end{array}$ & $\begin{array}{c}\text { MIC }_{90} \\
\text { range }(\mathrm{mg} / \mathrm{l})\end{array}$ \\
\hline \multicolumn{4}{|l|}{ Mycobacterium abscessus } \\
\hline TETS $^{S}$ & $\leq 0,06-1$ & $\leq 0,13$ & 0,25 \\
\hline $\mathrm{TET}^{\mathrm{R}}$ & $\leq 0,06-1$ & $\leq 0,13$ & 0,25 \\
\hline \multicolumn{4}{|l|}{ Mycobacterium chelonae } \\
\hline TETS $^{S}$ & $\leq 0,25$ & $\leq 0,06$ & $\leq 0,13$ \\
\hline TETR $^{\mathrm{R}}$ & $\leq 0,25$ & $\leq 0,06$ & $\leq 0,13$ \\
\hline \multicolumn{4}{|l|}{ Mycobacterium fortuitum gruppo } \\
\hline TETS & $\leq 0,25$ & $\leq 0,06$ & $\leq 0,13$ \\
\hline $\mathrm{TET}^{\mathrm{R}}$ & $\leq 0,25$ & $\leq 0,06$ & $\leq 0,13$ \\
\hline Mycobacterium avius complex & $\geq 32$ & $>32$ & $>32$ \\
\hline Mycobacterium lentiflavum & $\geq 32$ & $>32$ & $>32$ \\
\hline Mycobacterium marinum & $0,19-24$ & $2-16$ & $3-16$ \\
\hline Mycobacterium kansasii & $8-32$ & 16 & 32 \\
\hline Chlamydophilia pneumoniae & $0,13-0,25$ & 0,13 & 0,13 \\
\hline \multicolumn{4}{|l|}{ Mycoplasma hominis } \\
\hline TETS $^{S}$ & $0,13-0,5$ & 0,25 & 0,5 \\
\hline TET $^{\mathrm{R}}$ & $0,13-0,5$ & 0,25 & 0,5 \\
\hline Mycoplasma pneumoniae & $0,06-0,25$ & 0,13 & 0,25 \\
\hline Ureaplasma urealyticum & $1-16$ & 4 & 8 \\
\hline
\end{tabular}

\section{Tabella IV}

Attività in vitro di tigeciclina contro patogeni atipici (modificato da [14])

$\mathrm{R}=$ Resistente $\mathrm{S}=$ Suscettibile $\mathrm{TET}=$ Tetraciclina che tigeciclina si distribuisce rapidamente nei tessuti. Negli studi di farmacologia clinica in cui è stato usato il regime di dosaggio terapeutico di $100 \mathrm{mg}$ seguito da $50 \mathrm{mg}$ ogni 12 ore, $\mathrm{la}_{\text {max }}$ sierica di tigeciclina allo steady state è stata di $866+233 \mathrm{ng} / \mathrm{ml}$ per un'infusione di 30 minuti e di $634+97 \mathrm{ng} / \mathrm{ml}$ per un'infusione di 60 minuti. L'AUC 0-12h allo steady state è stata di $2349+$ $850 \mathrm{ng} / \mathrm{ml} \times \mathrm{h}$ [22].
In uno studio che ha valutato la distribuzione del farmaco nel siero, nei tessuti e nei liquidi corporei, una singola dose di $100 \mathrm{mg}$ ev ha prodotto concentrazioni considerevolmente alte nella bile, nella colecisti, nel colon e nel polmone in rapporto al siero. Inoltre la quantità di tigeciclina riscontrata nel tessuto osseo, nel liquido sinoviale e nel liquido cerebrospinale è risultata tra l'11 e il $41 \%$ delle concentrazioni

\begin{tabular}{|c|c|c|c|c|c|c|c|c|c|c|}
\hline & Staphylococcus & Streptococcus & Enterococchi & MRSA & VRE & Gram - & $\begin{array}{l}\text { Gram- } \\
\text { ESBL }\end{array}$ & Anaerobi & Atipici* & P. aeruginosa \\
\hline \multicolumn{11}{|l|}{ Tigeciclina } \\
\hline \multicolumn{11}{|l|}{ Ceftriaxone } \\
\hline \multicolumn{11}{|l|}{ Ceftazidime } \\
\hline \multicolumn{11}{|c|}{ Carbapenem§ } \\
\hline \multicolumn{11}{|l|}{ Levofloxacina } \\
\hline \multicolumn{11}{|l|}{$\begin{array}{l}\text { B-latt./inib. } \\
\text { B-lattamasi }\end{array}$} \\
\hline \multicolumn{11}{|l|}{ Linezolid } \\
\hline \multicolumn{11}{|l|}{$\begin{array}{l}\text { Quinupristin/ } \\
\text { dalfopristin }\end{array}$} \\
\hline \multicolumn{11}{|l|}{ Glicopeptidi } \\
\hline \multicolumn{11}{|l|}{ Doxiciclina } \\
\hline Minociclina & & & & & & & & & & \\
\hline
\end{tabular}

\section{Tabella V}

Spettro di attività di tigeciclina in confronto ad altri antibiotici

$\square=$ Non attivo; $\square=$ Attività intermedia o solo di alcuni antibiotici della classe; $\square=$ Attivo

*=Micoplasma, clamidia, micobatteri non tubercolari; **=Amoxicillina + clavulanato, ampicillina + sulbactam; $\S=$ Imipenem e meropenem 


\begin{tabular}{|c|c|c|c|}
\hline $\begin{array}{c}\text { Tessuto o } \\
\text { liquido corporeo }\end{array}$ & $\begin{array}{c}\text { AUC }_{0-24} \text { tessuto } \\
(\mathrm{mg} \mathrm{x} \mathrm{h/l} 0 \mathrm{mg} \mathrm{h} / \mathrm{kg})^{*}\end{array}$ & $\begin{array}{c}\text { AUC }_{0-24} \text { sierica } \\
(\mathrm{mg} \times \mathrm{h} / \mathrm{l})\end{array}$ & $\begin{array}{l}\text { Rapporto } \text { AUC }_{0-24} \\
\text { (tessuto/siero) }\end{array}$ \\
\hline Bile & $2.815 / 1.787$ & $5,24 / 4,86$ & $537 / 368$ \\
\hline Colecisti & $119,99 / 65,96$ & $5,24 / 4,86$ & $23 / 14$ \\
\hline Colon & $17,30 / 9,83$ & $6,58 / 5,46$ & $2,6 / 1,8$ \\
\hline Polmone & $9,19 / 8,02$ & $4,48 / 3,99$ & $2,0 / 2,0$ \\
\hline Osso & $2,05 / 1,26$ & $4,95 / 4,49$ & $0,41 / 0,28$ \\
\hline Liquido sinoviale & $1,68 / 1,58$ & $5,35 / 4,86$ & $0,31 / 0,32$ \\
\hline $\begin{array}{l}\text { CSF (liquido } \\
\text { cerebrospinale) }\end{array}$ & $0,460 / 0,426$ & $4,18 / 3,59$ & $0,11 / 0,12$ \\
\hline
\end{tabular}

\section{Tabella VI}

Distribuzione di tigeciclina nel siero, nei tessuti e nei liquidi corporei (rielaborata da [23])

$\mathrm{AUC}_{0-24}=$ area sotto la curva concentrazione-tempo da 0 a $24 \mathrm{~h}$; dati espressi come rapporto dei valori di concentrazione media/mediana rispetto ai tempi

* I dati espressi come mg x h/l comprendono bile, liquido sinoviale e CSF (liquido cerebrospinale). I dati espressi come $\mathrm{mg} \times \mathrm{h} / \mathrm{kg}$ comprendono colecisti, colon, polmone e bile

sieriche [23]. La Tabella VI riporta i dati più rilevanti dello studio.

\section{Metabolismo ed eliminazione}

La via primaria di eliminazione per tigeciclina è l'escrezione biliare sotto forma di tigeciclina non modificata. La glucoronizzazione e l'escrezione urinaria della tigeciclina non modificata sono vie di eliminazione secondarie. Nessun metabolita attivo è stato identificato. Il valore di emivita di eliminazione varia da $38 \mathrm{~h}$, dopo somministrazione singola di $100 \mathrm{mg}$, a $67 \mathrm{~h}$ allo steady state dopo somministrazione di $100 \mathrm{mg} / 12 \mathrm{~h}$ [24].

Il profilo farmacocinetico di tigeciclina dopo dose singola non è risultato modificato in pazienti con insufficienza epatica lieve mentre la clearance sistemica di tigeciclina è risultata ridotta del $25 \%$ e del $55 \%$ e l'emivita è risultata prolungata del $23 \%$ e del $43 \%$ in pazienti con insufficienza epatica rispettivamente moderata o grave (Child Pugh B e C). Tuttavia tali differenze non comportano alcun aggiustamento posologico nei pazienti con insufficienza epatica lieve e moderata mentre in caso di insufficienza epatica grave (Child Pugh C) la dose di tigeciclina deve essere ridotta a $25 \mathrm{mg}$ ogni 12 ore dopo una dose di carico di $100 \mathrm{mg}$.

Il recupero della radioattività totale nelle feci e nelle urine in seguito alla somministrazione di ${ }^{14} \mathrm{C}$-tigeciclina indica che il $59 \%$ della dose è eliminata tramite escrezione biliare/fecale mentre il $33 \%$ è escreto nelle urine. La clearance totale della tigeciclina è di $24 \mathrm{l} / \mathrm{h}$ dopo infusione endovenosa. La clearance renale è approssimativamente il $13 \%$ della clearance totale [22].

Studi in vitro indicano che tigeciclina non inibisce il metabolismo mediato dal citocromo P450 e, attualmente, non risultano interazioni con altri farmaci.

\section{EFFICACIA CLINICA}

L'efficacia di tigeciclina è stata analizzata in quattro studi principali di fase 3 .

Due di questi sono stati condotti su 1.642 pazienti con infezioni intra-addominali complicate e hanno messo a confronto tigeciclina con imipenem/cilastatina. Sono stati pubblicati i dati relativi ai due studi aggregati [25].

Gli altri due studi, che sono stati condotti su 1.116 pazienti con infezioni complicate della cute e dei tessuti molli, hanno confrontato tigeciclina con una combinazione di vancomicina e aztreonam. Anche in questo caso sono stati pubblicati i dati aggregati [26].

Tutti gli studi hanno misurato la risposta clinica (guarigione dall'infezione o fallimento della cura).

I risultati di questi studi hanno dimostrato che, nel trattamento di pazienti affetti da cIAI, l'efficacia clinica di $50 \mathrm{mg}$ di tigeciclina somministrati tramite infusione in monoterapia ogni 12 ore (dopo la dose iniziale di $100 \mathrm{mg}$ ) è statisticamente non inferiore a quella di un regime antibiotico con imipenem/cilastatina (dose da 200 a $500 \mathrm{mg}$ adeguata in base al peso del paziente e al valore della creatinina), somministrato per infusione endovenosa ogni 6 ore.

L'endpoint primario degli studi nelle infezioni intra-addominali complicate è stata la risposta clinica alla visita del test-of-cure (12-42 giorni dopo la terapia) negli endpoint co-primari delle popolazioni microbiologicamente valutabile (ME) e con intent to treat microbiologico modificato (m-mITT). Nella popolazione ME la percentuale di guarigione è stata dell' $86,1 \%$ (441/512) nel gruppo trattato con tigeciclina verso $86,2 \%(442 / 513)$ nel gruppo trattato con imipenem/cilastatina (95\% intervallo di confidenza (CI), da $-4.5 \%$ a $4.4 \%$; $<<0,0001$ per la non inferiorità). La percentuale di guarigione per la popolazione m-mITT è stata del $80,2 \%$ $(506 / 631)$ nel gruppo trattato con tigeciclina 
verso $81,5 \%$ (514/631) nel gruppo trattato con imipenem/cilastatina (95\% intervallo di confidenza, da $-5,8 \%$ a 3,2\%; $<<0,0001$ per la non inferiorità).

Pertanto tigeciclina ha soddisfatto, in conformità ai requisiti richiesti dalle autorità regolatorie europee, i criteri statistici di non inferiorità rispetto alla terapia imipenem/cilastatina per la risposta clinica (guarigione/fallimento) all'interno di ciascuna popolazione co-primaria (popolazioni valutabili microbiologicamente e popolazioni con intent to treat microbiologico modificato).

Per quanto riguarda il trattamento dei pazienti affetti da cSSSI, i risultati di questi studi hanno dimostrato che l'efficacia clinica di 50 $\mathrm{mg}$ di tigeciclina somministrati in monoterapia tramite infusione ev ogni 12 ore (dopo la dose iniziale di $100 \mathrm{mg}$ ), è statisticamente comparabile a quella di un regime antibiotico combinato di $1 \mathrm{~g}$ di vancomicina e $2 \mathrm{~g}$ di aztreonam somministrati per infusione ev ogni 12 ore.

In questi studi sui pazienti con infezioni complicate della cute e dei tessuti molli un totale di 1.116 pazienti costituisce la popolazione intent to treat modificato (m-ITT), mentre 1.057 pazienti (538 trattati con tigeciclina e 519 trattati con vancomicina + aztreonam) rappresentano la popolazione ITT clinico modificato (c-mITT) e 833 pazienti (422 trattati con tigeciclina e 411 trattati con vancomicina + aztreonam) rappresentano la popolazione clinicamente valutabile (CE). La risposta clinica a tigeciclina e a vancomicina + aztreonam alla fine della terapia è risultata simile: $\mathrm{c}-\mathrm{mITT}=79,7 \%(95 \%$ intervallo di confidenza $(\mathrm{CI})=76,1-83,1 \%$ ) verso $81,9 \%(95 \% \mathrm{CI}=78,3-85,1 \% ; \mathrm{p}=0,4183)$; clinicamente valutabile (CE) $86,5 \%(95 \% \mathrm{CI}$ $=82,9-89,6 \%)$ verso $88,6 \%(95 \% \mathrm{CI}=85,1-$ $91,5 \% ; \mathrm{p}=0,4233)$.

Tigeciclina ha soddisfatto anche in questo caso, in conformità ai requisiti richiesti dalle autorità regolatorie europee, $\mathrm{i}$ criteri statistici di non inferiorità alla terapia combinata vancomicina + aztreonam per la risposta clinica (guarigione/fallimento) all'interno di ciascuna popolazione co-primaria (popolazioni valutabili clinicamente e popolazioni con intent to treat clinico modificato).

In tutte le situazioni, tigeciclina è risultata efficace nel trattamento di infezioni causate da un ampio spettro di patogeni, osservati comunemente in pazienti affetti da cSSSI e cIAI, compresi i batteri dotati di elevata resistenza agli antibiotici comunemente indicati per queste infezioni.

I risultati emersi dagli studi clinici nel trattamento delle infezioni complicate della cute e dei tessuti molli (cSSSI) e delle infezioni complicate intra-addominali (cIAI) confermano in vivo l'efficacia microbiologica di questo antibiotico in grado di esprimere la sua attività nei confronti non solo di batteri sensibili (gram- positivi, gram-negativi e anaerobi) ma anche verso batteri che esprimono resistenza ad altri antibiotici, come gli MRSA e i produttori di ESBL.

Dal punto di vista clinico, tigeciclina potrebbe quindi rappresentare un'alternativa all'impiego dei farmaci attualmente in uso, poiché potrebbe da un lato fornire un'arma terapeutica in più, e dall'altro permettere una minore "pressione selettiva" da parte degli antibiotici già in uso e quindi un recupero della sensibilità dei batteri nei loro confronti.

\section{SICUREZZA}

I dati sulla sicurezza sono stati ricavati da un totale di 2.797 soggetti: 1.415 , che avevano ricevuto almeno una dose di tigeciclina nelle sperimentazioni di fase 3 , sono stati trattati con tigeciclina, e 1.382 sono stati trattati con farmaci di confronto [27].

Sono stati arruolati più uomini che donne (circa il $62 \%$ vs il $38 \%$, rispettivamente). La distribuzione dell'età era simile tra i due gruppi di trattamento, con un'età media di 47 anni. Circa il 19\% dei soggetti aveva un'età maggiore o uguale a 65 anni; circa 1' $8 \%$ aveva un'età maggiore o uguale a 75 anni. Circa il $65 \%$ dei soggetti era di razza bianca, il 7\% di razza nera e la restante asiatica o altro. Le caratteristiche demografiche e di base erano simili per entrambi i gruppi $(\mathrm{p}>0,05)$.

\section{Eventi avversi}

Gli eventi avversi treatment-emergent (TEAEs) sono stati definiti come gli eventi avversi non presenti durante la fase attiva (di trattamento) dello studio e che non rappresentavano una condizione cronica della storia medica del soggetto, oppure presenti all'inizio dello studio come parte della storia medica del soggetto ma la cui gravità o frequenza erano aumentate durante la fase attiva dello studio.

I TEAEs sono stati osservati nel $71,6 \%$ dei soggetti nel gruppo tigeciclina e nel $67,4 \%$ dei soggetti trattati con il farmaco di confronto $(\mathrm{p}=$ $0,017)$. I TEAEs correlati al farmaco più comuni nei pazienti trattati con tigeciclina sono stati: nausea nel 20,4\% (12,9\% lieve, 6,6\% moderata e $0,8 \%$ grave) e vomito nel $13,5 \%$ dei casi $(8,3 \%$ lieve, $4,5 \%$ moderata e $0,6 \%$ grave).

L'interruzione di tigeciclina è stata associata molto frequentemente alla nausea $(1,3 \%)$ e al vomito $(1,0 \%)$.

Nessuna conclusione può essere evidenziata dall'uso di terapia profilattica antiemetica a causa dell'esiguo numero di pazienti che hanno ricevuto terapia antiemetica profilattica prima della somministrazione di tigeciclina.

Le reazioni di ipersensibilità sono state raramente riferite con tetracicline e includono una ampia varietà di sintomi (per esempio, irritazione cutanea, dermatite, urticaria, prurito, 
anafilassi, febbre, emicrania, artralgia, ecc...). Alcuni TEAEs compatibili con reazioni di ipersensibilità sono stati riportati in pazienti trattati con tigeciclina. Ciò può essere complicato dal noto potenziale di rilascio dell'istamina.

\section{Eventi avversi gravi (SAEs)}

Un totale di 188 pazienti trattati con tigeciclina (pari al 13,3\%) e 159 pazienti trattati con il farmaco di confronto (1'11,5\%) hanno presentato uno o più SAEs in tutti gli studi di fase 3 (300, 305, 301, 306, 307 e 309). L'incidenza totale di SAEs era simile tra i due gruppi di trattamento $(p=0,169)$.

\section{INDICAZIONI APPROVATE}

Tigeciclina è indicata per il trattamento delle seguenti infezioni:
- infezioni complicate della cute e dei tessuti molli;

- infezioni complicate intra-addominali.

Le infezioni della cute e dei tessuti molli (SSSI) includono un vasto numero di condizioni cliniche che diventano complicate (cSSSI) quando sono presenti condizioni di co-morbilità che possono inficiare la risposta al trattamento, quando è richiesto un intervento chirurgico oppure quando avviene una progressione dagli strati superficiali della cute fino alle strutture più profonde.

Le complicazioni delle infezioni della cute e dei tessuti molli (cSSSI) possono essere causate da diversi meccanismi, come un peggioramento delle condizioni di una preesistente lesione della cute, acutizzazione di trauma, morsi o procedure operatorie, o cronicizzazione di ferite, come ad

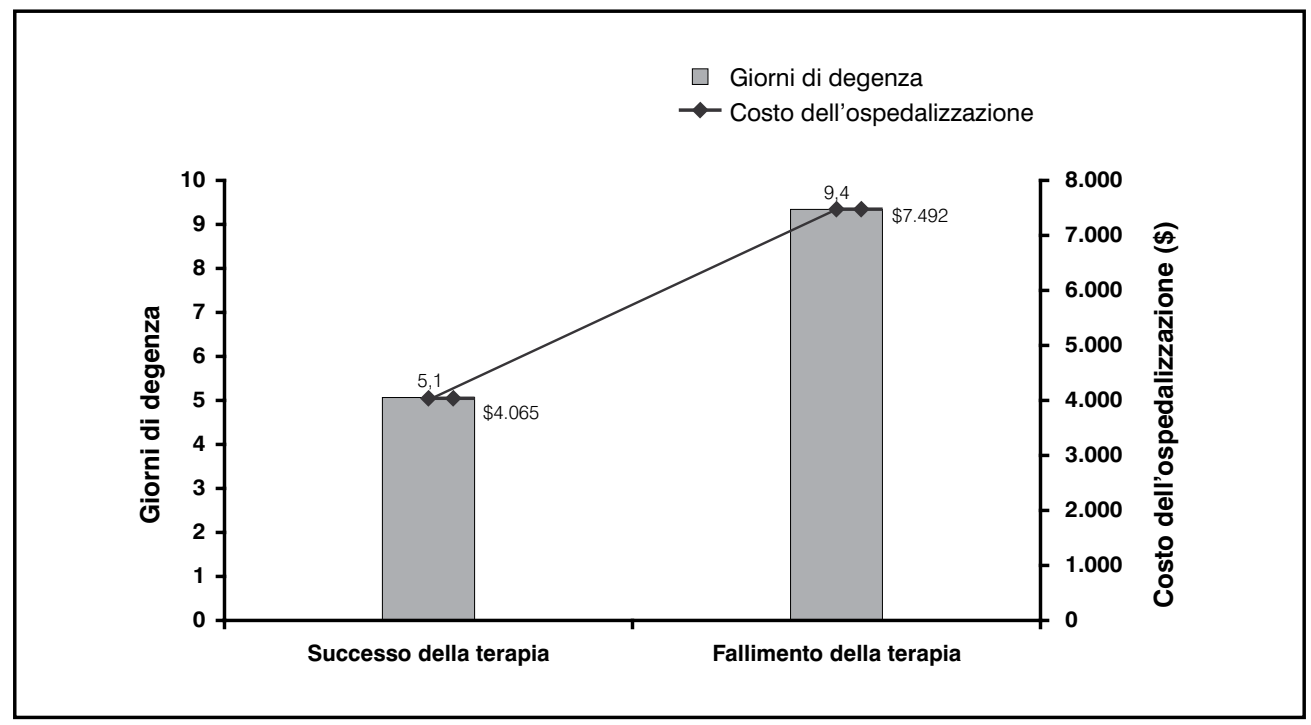

Figura 2

Durata della degenza e costi dell'ospedalizzazione nelle cSSSI (modificata da [38,39])

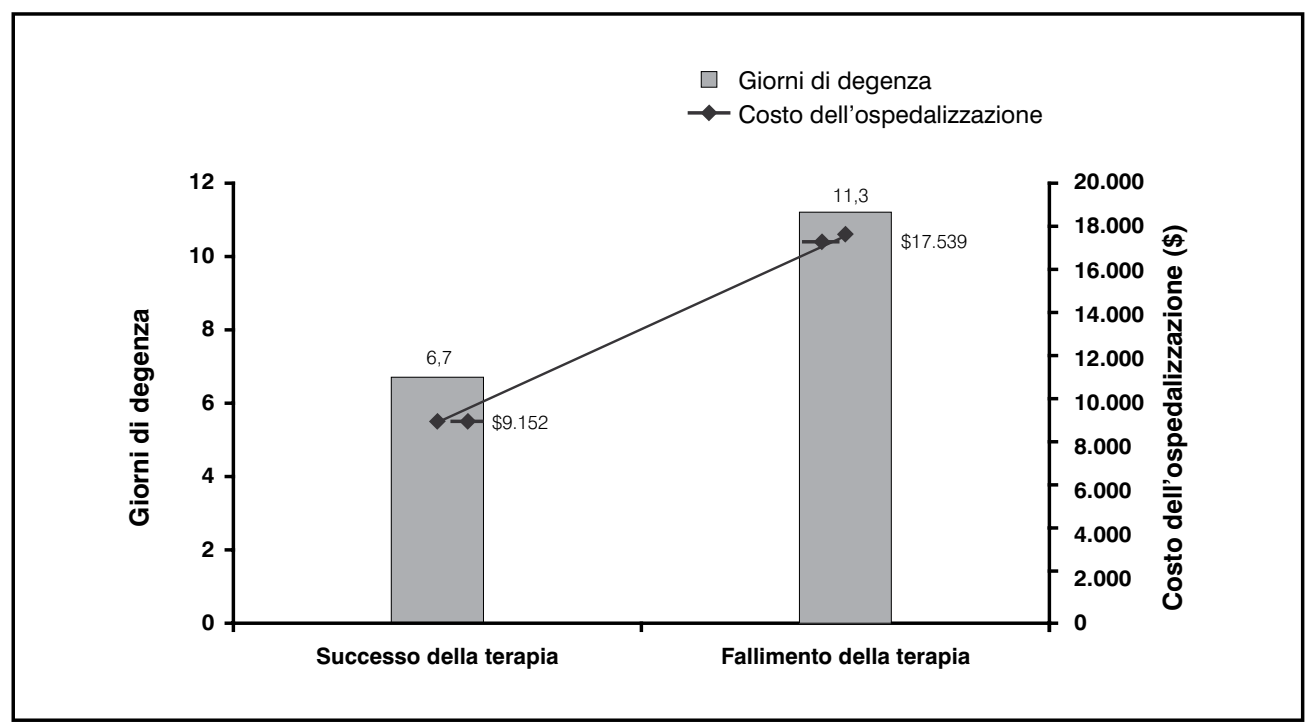

Figura 3

Durata della degenza e costi dell'ospedalizzazione nelle clAl (modificata da [40]) 
esempio ulcere del piede diabetico, piaghe da decubito o ulcere da stasi venosa.

Le infezioni intra-addominali (cIAI) sono definite come infezioni che si diffondono dal punto di origine allo spazio peritoneale mediante la diffusione attraverso parete intestinale o perforazione e sono associate a formazione di ascesso o peritonite.

\section{Dati epidemiologici}

Le infezioni richiedono necessariamente il ricovero del paziente in ospedale e sono la principale causa di prolungamento dell'ospedalizzazione e di morte in tutto il mondo [28]. È difficile quantificare il numero esatto di infezioni causate da patogeni resistenti in tutta Europa, a causa del diverso approccio dei sistemi di sorveglianza [29].

Si stima che in tutta l'Unione Europea ogni anno si verifichino 3 milioni di infezioni acquisite in ospedale, con il risultato di circa 50.000 morti attribuibili [30].

Circa il $60 \%$ di queste infezioni acquisite in ospedale sono causate da organismi resistenti agli antibiotici [31], e la percentuale di resistenza agli antibiotici sta raggiungendo livelli allarmanti in alcuni paesi europei [32].

Alcuni studi, di cui uno condotto dall'Organizzazione Mondiale della Sanità, hanno sottolineato che una terapia antibiotica inappropriata dovuta alla resistenza all'antibiotico porta a un incremento dei costi sanitari associati con un prolungamento della malattia, maggiore frequenza di ricoveri ospedalieri e prolungamento del periodo di ospedalizzazione [33-36]. L'uso di antibiotici ad ampio spettro è necessario per il trattamento empirico delle infezioni complicate, spesso polimicrobiche, causate da batteri non identificabili nelle 2448 ore e spesso da patogeni resistenti. Come è noto, un trattamento tempestivo e adeguato ha un impatto significativamente positivo sulla risoluzione dell'infezione e sulla riduzione del rischio di morte.

Oltre al costo in termini di vite umane, il fallimento del trattamento antibiotico determina un incremento dei costi ospedalieri.

Pazienti che sperimentano insuccessi della terapia iniziale richiedono infatti una maggiore durata del trattamento antibiotico parenterale o interventi chirurgici addizionali, il che spesso comporta un prolungamento dell'ospedalizzazione o un trasferimento in reparti di terapia intensiva, con conseguente incremento dei costi in termini di risorse umane e finanziarie [37].

In uno studio multicentrico condotto negli Stati Uniti è stato calcolato che la durata media della degenza ospedaliera per i pazienti con cSSSI che hanno ricevuto una terapia empirica iniziale efficace era di 5,1 giorni, mentre per i pazienti che hanno sperimentato un insuccesso del trattamento era di 9,4 giorni. Calcolando un costo ospedaliero giornaliero medio di 797 \$, il costo totale dell'ospedalizzazione incrementava da 4.065 \$ a $7.492 \$$ in seguito al fallimento della terapia empirica iniziale $[38,39]$.

Per le cIAI, è stato calcolato che il fallimento della terapia antibiotica empirica portava i giorni di degenza dei pazienti a 11,3 contro i 6,7 giorni dei pazienti che avevano ricevuto una terapia antibiotica empirica efficace. Il costo totale dell'ospedalizzazione incrementava dai $9.152 \$$ in caso di terapia antibiotica efficace ai $17.539 \$$ in caso di fallimento del trattamento [40].

\section{CONCLUSIONI}

La resistenza batterica agli antibiotici è diventata un serio problema per il trattamento delle infezioni ospedaliere, comprese le infezioni complicate cSSSI e cIAI, a causa del continuo incremento dei ceppi multiresistenti e della diffusione della resistenza crociata ai trattamenti antibiotici. Con l'emergenza di questo tipo di resistenze, l'efficacia degli antibiotici usati comunemente viene facilmente compromessa e può risultare inadeguata, sia per trattare le infezioni acquisite in ospedale sia per quelle acquisite in comunità che necessitano di ospedalizzazione.

Tigeciclina è indicata per il trattamento in monoterapia di pazienti affetti da infezioni complicate della cute e dei tessuti molli e di pazienti affetti da infezioni complicate intra-addominali, sia mono- che polimicrobiche, causate non solo da batteri comunemente sensibili ma anche da quelli contenenti geni responsabili di elevata resistenza agli antibiotici, come i batteri produttori di beta-lattamasi a spettro esteso (ESBL) e $S$. aureus meticillino-resistente (MRSA).

Tigeciclina, disponibile in formulazione iniettabile, è un antibiotico appartenente alla classe delle glicilcicline, in grado di superare due dei principali meccanismi di resistenza batterica alle vecchie tetracicline: la protezione ribosomiale e le pompe di efflusso. La presenza della catena laterale glicilica, in posizione 9 nell'anello D, conferisce alla molecola base, derivata dalla minociclina, una peculiare caratteristica che le permette di superare tali meccanismi di resistenza e di allargare lo spettro d'azione della molecola. Inoltre, tigeciclina sfugge ad altri meccanismi di resistenza come la produzione di $\beta$-lattamasi a spettro esteso (ESBL, Extended Spectrum Beta-Lactamases) che può limitare la scelta degli antibiotici disponibili.

L'introduzione di tigeciclina fornisce al clinico un'alternativa terapeutica in più, non solo in funzione dell'attività sui batteri causa di infezione nei singoli pazienti, ma anche permettendo di utilizzare una classe di antibiotici diversa rispetto a quelle attualmente disponibili e di conseguenza consentire una minore pressione selettiva da parte di altri antibiotici comunemente riconosciuti come forti indut- 


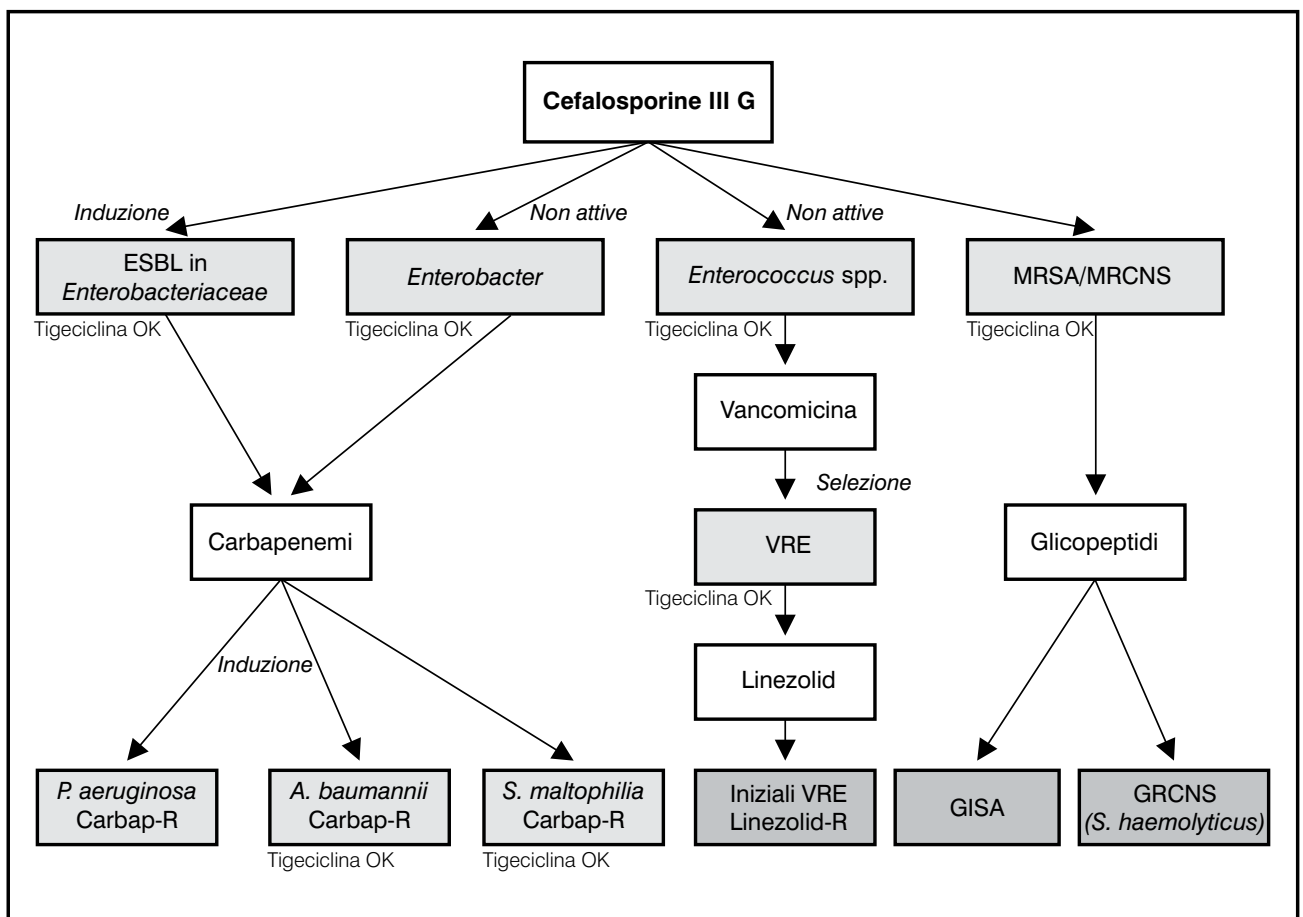

Figura 4

Processi di selezione e induzione di resistenze batteriche

tori di resistenza. Un trattamento tempestivo e adeguato ha un impatto significativamente positivo sulla risoluzione delle infezioni gravi e sulla riduzione del rischio di morte nei pazienti critici: oltre al costo in termini di vite umane, il fallimento del trattamento antibiotico determina un incremento dei costi ospedalieri.

Come si evince dalla Figura 4, tigeciclina può essere utilizzata come alternativa a diverse classi di antibiotici (carbapenemi, linezolid, glicopeptidi) spesso usate in associazione allo scopo di garantire alla terapia empirica un ampio spettro di copertura. Tigeciclina ha in effetti caratteristiche di efficacia e sicurezza in monoterapia che permettono di evitare l'associazione con altri antibiotici nel trattamento delle infezioni complicate cSSSI e cIAI. Tigeciclina si può utilizzare nel paziente adulto sempre con lo stesso dosaggio senza necessità di ridurre la posologia in base al peso, alla razza, al sesso, alla funzionalità renale o epatica (aggiustamento posologico solo per insufficienza epatica grave Child Pugh C) o a patologie concomitanti.

\section{BIBLIOGRAFIA}

1. Goossens H, Ferech M, Stichele RV et al and the ESAC Project Group. Outpatient antibiotic use in Europe and association with resistance; a cross-national database study. Lancet 2005; 365: 579-587

2. Shlaes DM, Gerding DN, John JF et al. Society for Healthcare Epidemiology of America and Infectious Diseases Society of America Joint Committee on the Prevention of Antimicrobial Resistance: Guidelines for the prevention of antimicrobial resistance in hospitals. Clin Infect Dis 1997; 25: 584-599

3. Cosgrove SE, Carmeli Y. The impact of antimicrobial resistance on health and economic outcomes. Clin Infect Dis 2003; 36: 1433-1437

4. Connel SR, Tracz DM, Nierhaus KH et al. Ribosomal protection proteins and their mechanism of tetracycline resistance. Antimicrob Agents Chemother 2003; 47: 3675-81 
5. Petersen PJ, Jacobus NV, Weiss WJ et al. In vitro and in vivo antibacterial activities of a novel glycylcycline, the 9-t-butylglycylamido derivative of minocycline (GAR-936). Antimicrob Agents Chemother 1999; 43: 738-44

6. Projan SJ. Preclinical pharmacology of GAR-936, a novel glycylcycline antibacterial agent. Pharmacotherapy 2000; 20: 219S-28S

7. Bergeron J, Ammirati M, Danley D et al. Glycylcyclines bind to the high-affinity tetracycline ribosomal binding site and tet(M)- and tet(O)-mediated ribosomal protection. Antimicrob Agents Chemother 1996; 40: 2226-8

8. Baquero F. Gram-positive resistance: challenge for the development of new antibiotics. J Antimicrob Chemother 1997; 39(Suppl A): 1-6

9. Hoban DJ, Bouchillon SK, Johnson BM et al. In vitro activity of tigecycline against 6792 gram-negative and grampositive clinical isolates from the global Tigecycline Evaluation Surveillance Trial (TEST Program, 2004). Diagn Microbiol Infect Dis 2005; 52: 215-27

10. Bouchillon S, Stevens T, Johnson B et al. Tigecycline Evaluation Surveillance Trial (TEST) - global in vitro antibacterial activity against 13,669 gram-positive and gram-negative pathogens. Copenhagen: 15th European Congress of Clinical Microbiology and Infectious Diseases, 2005

11. Sader HS, Jones RN, Stilwell MG et al. Tigecycline activity tested against 26,474 bloodstream infection isolates: a collection from 6 continents. Diagn Microbiol Infect Dis 2005; 52: 181-6

12. Fritsche TR, Strabala PA, Sader HS et al. Activity of tigecycline tested against a global collection of Enterobacteriaceae, including tetracycline-resistant isolates. Diagn Microbiol Infect Dis 2005; 52: 209-13

13. Fritsche TR, Sader HS, Stilwell MG et al. Potency and spectrum of tigecycline tested against an international collection of bacterial pathogens associated with skin and soft tissue infections (2000-2004). Diagn Microbiol Infect Dis 2005; 52: 195-201

14. Pankey GA. Tigecycline. J Antimicrob Chemother 2005; 56, 470-480

15. Betriu C, Rodriguez-Avial I, Sanchez BA et al. Comparative in vitro activities of tigecycline (GAR-936) and other antimicrobial agents against Stenotrophomonas maltophilia (letter). J Antimicrob Chemother 2002; 50: 758-9

16. Pachon-Ibanez ME, Jimenez-Mejias ME, Pichardo C et al. Activity of tigecycline (GAR-936) against Acinetobacter baumannii strains, including those resistant to imipenem. Antimicrob Agents Chemother 2004; 48: 4479-81

17. Jacobus NV, McDermott LA, Ruthazer R et al. In vitro activities of tigecycline against the Bacteroides fragilis group. Antimicrob Agents Chemother 2004; 48: 1034-6

18. Edlund C, Nord CE. In-vitro susceptibility of anaerobic bacteria to GAR-936, a new glycylcycline. Clin Microbiol Infect 2000; 6: 159-63

19. Betriu C, Rodriguez-Avial I, Sanchez BA et al. Spanish Group of Tigecycline. In vitro activities of tigecycline (GAR-936) against recently isolated clinical bacteria in Spain. Antimicrob Agents Chemother 2002; 46: 892-5

20. Wallace RJ, Brown-Elliott BA, Crist CJ et al. Comparison of the in vitro activity of the glycylcycline tigecycline (formerly GAR-936) with those of tetracycline, minocycline, and doxycycline against isolates of nontuberculous mycobacteria. Antimicrob Agents Chemother 2002; 46: 3164-7

21. Roblin PM, Hammerschlag MR. In vitro activity of GAR-936 against Chlamydia pneumoniae and Chlamydia trachomatis. Int J Antimicrob Agents 2000; 16: 61-3

22. EMEA. Tygacil, Riassunto delle caratteristiche del prodotto, disponibile su www.emea.eu.int, accesso novembre 2006

23. Rodvold KA, Gotfried MH, Cwik M, Korth-Bradley JM, Dukart G, Ellis-Grosse EJ. Serum, tissue and body fluid concentrations of tigecycline after a single $100 \mathrm{mg}$ dose. J Antimicrob Chemother 2005; 49: 220-229

24. Muralidharan G, Micalizzi M, Speth J et al. Pharmacokinetics of tigecycline after single and multiple doses in healthy subjects. Antimicrob Agents Chemother 2005; 49: 220-229

25. Babinchak T, Ellis-Grosse E, Dartois N, Rose GM, Loh E and the Tigecycline 301 and 306 Study Groups. The efficacy and safety of tigecycline for the treatment of complicated intra-abdominal infections: analysis of pooled clinical trial data. Clin Infect Dis 2005; 41: 354-67

26. Ellis-Grosse E, Babinchak T, Dartois N, Rose G, Loh E and the Tigecycline 300 and 305 cSSSI Study Groups. The efficacy and safety of tigecycline in the treatment of skin and skin-structure infections: results of 2 double-blind phase 3 comparison studies with vancomycin-aztreonam. Clin Infect Dis 2005; 41: 341-53

27. EMEA/H/C/644, Tygacil, relazione di valutazione pubblica europea (EPAR), 2006 


\section{Tigeciclina: rassegna della letteratura}

28. World Health Organisation. Prevention of hospital-acquired infections: a practical guide. Disponibile su http://www. who.int/csr/resources/publications/drugresist/whocdscsreph200212.pdf

29. National Audit Office. A comparison of international practices in the management and control of hospital-acquired infections: report. 26 September 2003

30. Hospitals in Europe Link for Infection Control Through Surveillance (HELICS). About HELICS. Disponibile su http://helics.univ-lyon1.fr/helicshome.htm

31. World Health Organisation. Overcoming antimicrobial resistance: World Health Report on infectious diseases 2000. Disponibile su http:/www.who.int/infectious-disease-report/2000/other_versions/index-rpt2000_text.html

32. Goossens H, Ferech M, Stichele MV et al. Outpatient antibiotic use in Europe and association with resistance: a cross-national database study. Lancet 2005; 365: 579-87

33. Carmeli Y, Eliopoulos G, Mozaffari E et al. Health and economic outcomes of vancomycin-resistant enterococci. Arch Intern Med 2002; 162: 2223-8

34. Cosgrove SE, Kaye KS, Eliopoulos GM et al. Health and economic outcomes of the emergence of third-generation cephalosporin resistance in enterobacter species. Arch Intern Med 2002; 162: 185-90

35. Carmeli Y, Troillet N, Karchmer AW et al. Health and economic outcomes of antibiotic resistance in Pseudomonas aeruginosa. Arch Intern Med 1999; 159: 1127-32

36. World Health Organisation. WHO global strategy for containment of antimicrobial resistance. 2001. Disponibile su http://www.who.int/drugresistance/WHO_Global_Strategy_English.pdf

37. Cattan P, Yin DD, Sarfati E et al. Cost of care for inpatients with community-acquired intra-abdominal infections. Eur J Clin Microbiol Infect Dis 2002; 21: 787-93

38. Mallick R, Kuznik A. The expected economic burden of methicillin-resistant Staphylococcus aureus in complicated skin and skin structure infections. Abstract \#65; page 85. 16th Annual Meeting of the Society for Healthcare Epidemiology of America, 2006

39. Kuznik A, Mallick R. The expected economic burden of methicillin-resistant Staphylococcus aureus in complicated skin and skin structure infections - a modelling approach. Poster P1493. 16th European Congress of Clinical Microbiology and Infectious Diseases, 2006

40. Berger A, Edelsberg J, Schell S et al. Clinical and economic consequences of initial antibiotic therapy failure in complicated intra-abdominal infections. Page 258. Infectious Diseases Society of America. 43rd Annual Meeting Proceedings, 2005 